PROCEEDINGS OF THE

AMERICAN MATHEMATICAL SOCIETY

Volume 129, Number 1, Pages 59-67

S 0002-9939(00)05523-4

Article electronically published on June 21, 2000

\title{
A CHARACTERIZATION OF ALGEBRAS WITH POLYNOMIAL GROWTH OF THE CODIMENSIONS
}

\author{
A. GIAMBRUNO AND M. ZAICEV
}

(Communicated by Ken Goodearl)

\begin{abstract}
Let $A$ be an associative algebras over a field of characteristic zero. We prove that the codimensions of $A$ are polynomially bounded if and only if any finite dimensional algebra $B$ with $\operatorname{Id}(A)=\operatorname{Id}(B)$ has an explicit decomposition into suitable subalgebras; we also give a decomposition of the $n$-th cocharacter of $A$ into suitable $S_{n}$-characters.

We give similar characterizations of finite dimensional algebras with involution whose $*$-codimension sequence is polynomially bounded. In this case we exploit the representation theory of the hyperoctahedral group.
\end{abstract}

\section{$\S 1$. INTRODUCTION}

Let $F$ be a field of characteristic zero and $F\langle X\rangle=F\left\langle x_{1}, x_{2}, \ldots\right\rangle$ the free algebra of countable rank over $F$. If $A$ is a PI-algebra over $F$, that is, an algebra satisfying a polynomial identity, we let $\operatorname{Id}(A)$ be the T-ideal of $F\langle X\rangle$ of identities of $A$. It is well known that $\operatorname{Id}(A)$ is completely determined by the multilinear polynomials it contains; if $V_{n}=\operatorname{Span}\left\{x_{\sigma(1)} \cdots x_{\sigma(n)} \mid \sigma \in S_{n}\right\}$ is the space of multilinear polynomials in $x_{1}, \ldots, x_{n}$, then the sequence $c_{n}(A)=\operatorname{dim}_{F} \frac{V_{n}}{V_{n} \cap \operatorname{Id}(A)}, n=1,2, \ldots$, is called the sequence of codimensions of $A$ and it is an important numerical invariant of $\operatorname{Id}(A)$.

It was proved by Regev in [ $[\mathrm{R}]$ that for any PI-algebra $A, c_{n}(A)$ is exponentially bounded, i.e., there exist constants $a, \alpha>0$ such that $c_{n}(A) \leq a \alpha^{n}$ for all $n$.

In this paper we study algebras $A$ whose codimension sequence is polynomially bounded i.e., such that for all $n, c_{n}(A) \leq a n^{t}$ for some constants $a, t$. Kemer in K1 gave a characterization of such T-ideals in the language of the representation theory of $S_{n}$. It also follows from $\mathrm{K} 2$ that if $c_{n}(A)$ is polynomially bounded, then $I d(A)=I d(B)$ for a suitable finite dimensional algebra $B$.

For any finite dimensional algebra $A$ over an algebraically closed field we shall prove that $A$ has polynomial growth of the codimensions if and only if $A=A_{0} \oplus A_{1} \oplus$ $\cdots \oplus A_{m}$ where $A_{0}, A_{1}, \ldots, A_{m}$ are $F$-algebras such that 1) for $i=1, \ldots, m, A_{i}=$ $B_{i}+J_{i}$, where $B_{i} \cong F$ and $J_{i}$ is a nilpotent ideal of $\left.A_{i}, 2\right) A_{0}, J_{1}, \ldots, J_{m}$ are nilpotent right ideals of $A$ and 3) $A_{i} A_{k}=0$ for all $i, k \in\{1, \ldots, m\}, i \neq k$ and $B_{i} A_{0}=0$.

Received by the editors December 1, 1998 and, in revised form, March 26, 1999.

1991 Mathematics Subject Classification. Primary 16R10, 16R50; Secondary 16P99.

The first author was partially supported by the CNR and MURST of Italy; the second author was partially supported by RFFI, grants 96-01-00146 and 96-15-96050. 
Another description of such algebras is given, as in Kemer's paper [K1], in the language of the cocharacters as follows. The symmetric group $S_{n}$ acts on the left on $V_{n}$ by $\sigma f\left(x_{1}, \ldots, x_{n}\right)=f\left(x_{\sigma(1)}, \ldots, x_{\sigma(n)}\right), \sigma \in S_{n}, f\left(x_{1}, \ldots, x_{n}\right) \in V_{n}$. This action induces a structure of left $S_{n}$-module on $\frac{V_{n}}{V_{n} \cap I d(A)}$ and we write $\chi_{n}(A)$ for its $S_{n}$-character; $\chi_{n}(A)$ is called the $n$-th cocharacter of $A$. Let $\chi_{\lambda}$ denote the irreducible $S_{n}$-character associated to the partition $\lambda=\left(\lambda_{1}, \ldots, \lambda_{t}\right) \vdash n$ and write $\chi_{n}(A)=\sum_{\lambda \vdash n} m_{\lambda} \chi_{\lambda}$ where $m_{\lambda} \geq 0$ are the corresponding multiplicities. We shall prove that if $\operatorname{dim}_{F} A<\infty, A$ has polynomial growth of the codimensions if and only if

$$
\chi_{n}(A)=\sum_{\substack{\lambda \vdash n \\|\lambda|-\lambda_{1}<q}} m_{\lambda} \chi_{\lambda}
$$

where $J$ is the Jacobson radical of $A$ and $J^{q}=0$.

In the second part of the paper we address ourself to similar questions in the setting of algebras with involution. Let $F\langle X, *\rangle=F\left\langle x_{1}, x_{1}^{*}, x_{2}, x_{2}^{*}, \ldots\right\rangle$ be the free algebra with involution $*$ and $V_{n}(*)$ the space of multilinear $*$-polynomials in $x_{1}, x_{1}^{*}, \ldots, x_{n}, x_{n}^{*}$. For an algebra with involution $A$ we let $\operatorname{Id}(A, *)$ be the ideal of *-identities of $A$; then the sequence of $*$-codimensions is

$$
c_{n}(A, *)=\operatorname{dim}_{F} \frac{V_{n}(*)}{V_{n}(*) \cap \operatorname{Id}(A, *)}, n=1,2, \ldots .
$$

In the last section we characterize finite dimensional algebras $A$ such that $c_{n}(A, *)$ is polynomially bounded. Moreover by using the representation theory of the hyperoctahedral group $\mathbb{Z}_{2} \sim S_{n}$, we shall obtain a characterization of $A$ in terms of the *-cocharacter sequence of $A$.

\section{§2. Algebras with polynomial growth of the codimensions}

In this section we shall characterize algebras with polynomial growth of the codimensions. The following reduction is due to Kemer.

Theorem 1. Let $A$ be a PI-algebra. If for all $n, c_{n}(A) \leq a n^{t}$ for some constants $a, t$, then there exists a finite dimensional algebra $B$ such that $\operatorname{Id}(A)=\operatorname{Id}(B)$.

Proof. Let $G$ be the Grassmann algebra of countable dimension over $F$. By $[\mathrm{KR}$, $c_{n}(G)=2^{n-1}$, hence, for $n$ large, $c_{n}(A)<c_{n}(G)$. This implies that $\operatorname{Id}(A) \nsubseteq I d(G)$ and, by a theorem of Kemer [K2, Theorem 2.3] there exists a finite dimensional algebra $B$ such that $\operatorname{Id}(A)=\operatorname{Id}(B)$.

We remark that $c_{n}(A)$ does not change upon extension of the ground field $F$. In fact, if $K$ is an extension field of $F$, then $\operatorname{Id}(A) \otimes_{F} K=\operatorname{Id}\left(A \otimes_{F} K\right)$. Therefore in studying properties of $c_{n}(A)$ we may as well assume that $F$ is an algebraically closed field.

Theorem 2. Let $A$ be a finite dimensional algebra over an algebraically closed field $F$. Then the sequence of codimensions $\left\{c_{n}(A)\right\}_{n \geq 1}$ is polynomially bounded if and only if

1) $A=A_{0} \oplus A_{1} \oplus \cdots \oplus A_{m}$ a vector space direct sum of $F$-algebras where for $i=$ $1, \ldots, m, A_{i}=B_{i}+J_{i}, B_{i} \cong F, J_{i}$ a nilpotent ideal of $A_{i}$ and $A_{0}, J_{1}, \ldots, J_{m}$ are nilpotent right ideals of $A$;

2) for all $i, k \in\{1, \ldots, m\}, i \neq k, A_{i} A_{k}=0$ and $B_{i} A_{0}=0$. 
Proof. Let $A=B+J$ be the Wedderburn-Malcev decomposition of $A$ (CR) Theorem 72.19]) where $B$ is a semisimple subalgebra of $A$ and $J=J(A)$ its Jacobson radical. Write $B=B_{1} \oplus \cdots \oplus B_{m}$ with $B_{1}, \ldots, B_{m}$ simple $F$-algebras. Since $c_{n}(A)$ is polynomially bounded, by [GZ, $B_{i} J B_{k}=0$ for all $i \neq k$ and $\operatorname{dim}_{F} B_{i}=1, i, k=1, \ldots, m$.

Let $e=e_{1}+\cdots+e_{m}$ be the decomposition of the unit element of $B$ into orthogonal central (in $B$ ) idempotents; thus $e_{i} B=B_{i} \cong F$. Define for all $i=$ $1, \ldots, m, J_{i}=e_{i} J$ and $J_{0}=\{x \in J \mid B x=0\}$. It is easy to show that $A=$ $B+J=\left(B_{1}+J_{1}\right) \oplus \cdots \oplus\left(B_{m}+J_{m}\right) \oplus J_{0}$; let $A_{i}=B_{i}+J_{i}$ and $A_{0}=J_{0}$.

For $i \neq k \in\{1, \ldots, m\}, A_{i} A_{k}=\left(B_{i}+J_{i}\right)\left(B_{k}+J_{k}\right)=0$ since $e_{i} e_{k}=0$ and $B_{i} J B_{k}=0$. Also, for $i \neq 0, B_{i} A_{0}=0$.

Viceversa, let $A$ satisfy 1 ) and 2). From the relations $A_{i} A_{k}=0$ and $B_{i} A_{0}=0$ it follows that $J=A_{0}+J_{1}+\cdots+J_{m}$ is a nilpotent two-sided ideal of $A$ and $A=B_{1} \oplus \cdots \oplus B_{m} \oplus J$ where $B_{i} \cong F$ for all $i$. Since from the defining relations $A_{i} A_{k}=0$ and $B_{i} A_{0}=0$ it follows that $B_{i} J B_{k}=0$ for all $i \neq k$, then $c_{n}(A)$ is polynomially bounded by [GZ].

As an immediate consequence of the above result we get

Corollary 1. Let $A$ be the algebra described in the previous theorem. Let $J$ be the Jacobson radical of $A$ and, for $i=1, \ldots, m$, let $C_{i}=A_{i} \oplus A_{0}$. Then

$$
\operatorname{Id}(A)=\operatorname{Id}\left(C_{1}\right) \cap \ldots \cap \operatorname{Id}\left(C_{m}\right) \cap \operatorname{Id}(J) .
$$

Proof. Let $f \in \operatorname{Id}\left(C_{1}\right) \cap \ldots \cap \operatorname{Id}\left(C_{m}\right) \cap \operatorname{Id}(J)$ and suppose that $f \notin \operatorname{Id}(A)$. We may clearly assume that $f$ is multilinear and let $r_{1}, \ldots, r_{s} \in A$ be such that $f\left(r_{1}, \ldots, r_{s}\right) \neq 0$.

If $r_{1}, \ldots, r_{s} \in J$, then $f \notin I d(J)$, a contradiction. Hence there exists $r_{i} \notin J$; by linearity we may assume that $r_{i} \in B_{k}$ for some $k$. Recall that, for all $l, B_{l} A_{0}=$ $0, J_{l}$ is a right ideal of $A$ and, in case $l \neq k, A_{k} A_{l}=A_{l} A_{k}=0$. From an easy calculation it follows that $r_{1}, \ldots, r_{i-1}, r_{i+1}, \ldots, r_{s} \in A_{k} \cup A_{0}$. But then $f \notin \operatorname{Id}\left(C_{k}\right)$, a contradiction.

What can be said if $F$ is not algebraically closed?

Let $A$ be a finite dimensional algebra over a field $F$ and write $A=B+J, B=$ $B_{1} \oplus \cdots \oplus B_{m}$ with the $B_{i}$ 's simple algebras. If $\bar{F}$ is the algebraic closure of $F$, we write $\bar{A}=A \otimes_{F} \bar{F}$; moreover, since $J(\bar{A})=J(A) \otimes_{F} \bar{F}$ (see [Ro Theorem 2.5.36]), we get that

$$
\bar{A} \cong \overline{B_{1}} \oplus \cdots \oplus \overline{B_{m}}+J(\bar{A})
$$

where $\overline{B_{i}}=B_{i} \otimes_{F} \bar{F}$ are semisimple algebras.

Let $Z\left(B_{i}\right)$ be the center of $B_{i}$ and $t_{i}=\operatorname{dim}_{F} Z\left(B_{i}\right)$. Then $\overline{B_{i}} \cong C_{i 1} \oplus \cdots \oplus C_{i t_{i}}$ where $C_{i 1} \cong \ldots \cong C_{i k}$ are central simple algebras over $\bar{F}$.

In case $c_{n}(A)=c_{n}(\bar{A})$ is polynomially bounded, by [GZ], $C_{i k} \cong \bar{F}$ for all $i, k$ and $C_{i k} J(\bar{A}) C_{u v}=0$ if $(i, k) \neq(u, v)$. It follows that for all $i=1, \ldots, m, B_{i}$ is a field extension of $F$ of degree $t_{i}$. Since $\operatorname{char} F=0$, we write $B_{i}=F\left(a_{i}\right)$, a simple algebraic extension of $F$ of degree $t_{i}$. We have shown that if $F$ is any field and $A$ is an $F$-algebra with polynomial growth of the codimensions, then

$$
A \cong F\left(a_{1}\right) \oplus \cdots \oplus F\left(a_{m}\right)+J(A)
$$

and for all $i \neq k, F\left(a_{i}\right) J(A) F\left(a_{k}\right)=0$. 
We next give a characterization of polynomial growth in terms of the cocharacter sequence of the algebra.

In the sequel for $\lambda \vdash n$ we also write $|\lambda|=n$. We write $\chi_{\lambda}(1)=d_{\lambda}$ for the degree of the irreducible $S_{n}$-character $\chi_{\lambda}$ and, if $T_{\lambda}$ is a tableau of shape $\lambda$, we let $e_{T_{\lambda}}$ be the corresponding essential idempotent of $F S_{n}$. Notice that if $\lambda=\left(\lambda_{1}, \lambda_{2}, \ldots\right) \vdash n$, then $|\lambda|-\lambda_{1}$ denotes the number of boxes below the first row of the diagram of $\lambda$.

Theorem 3. Let $A$ be a finite dimensional algebra over a field $F$. Then $\left\{c_{n}(A)\right\}_{n \geq 1}$ is polynomially bounded if and only if

$$
\chi_{n}(A)=\sum_{\substack{\lambda \vdash n \\|\lambda|-\lambda_{1}<q}} m_{\lambda} \chi_{\lambda}
$$

where $J(A)^{q}=0$.

Proof. Note that the decomposition of $\chi_{n}(A)$ into irreducible components does not change when extending the base field. Therefore, since $J\left(A \otimes_{F} \bar{F}\right)^{q}=0$, we may assume, without lost of generality, that $F$ is algebraically closed.

Suppose first that the codimensions of $A$ are polynomially bounded. Let $\lambda$ be a partition of $n$ such that $|\lambda|-\lambda_{1} \geq q$ and suppose by contradiction that $m_{\lambda} \neq 0$. Then there exists a tableau $T_{\lambda}$ such that $e_{T_{\lambda}}\left(x_{1}, \ldots, x_{n}\right) \notin \operatorname{Id}(A)$. Let $\lambda^{\prime}=\left(\lambda_{1}^{\prime}, \ldots, \lambda_{t}^{\prime}\right)$ be the conjugate partition of $\lambda$. Then $e_{T_{\lambda}}\left(x_{1}, \ldots, x_{n}\right)$ is a linear combination of polynomials each alternating on $t$ disjoint sets of $\lambda_{1}^{\prime}, \ldots, \lambda_{t}^{\prime}$ variables, respectively. We shall reach a contradiction by proving that each such polynomial $f$ vanishes in $A$.

Fix a basis of $A$ which is the union of bases of $B_{1}, \ldots, B_{m}$ and $J$ respectively. Since $B_{i} B_{k}=B_{i} J B_{k}=0$ for all $i \neq k$, in order to get a non-zero value of $f$ we must replace all the variables with elements of $J$ and of one simple component, say, $B_{i}$. Also, since $\operatorname{dim} B_{i}=1$, we can substitute at most one element of $B_{i}$ in each alternating set. Hence we can substitute in all at most $t=\lambda_{1}$ elements from $B_{i}$. It follows that in order to get a non-zero value, we must substitute at least $|\lambda|-\lambda_{1} \geq q$ elements from $J$. Since $J^{q}=0$, we get that $f \equiv 0$ and with this contradiction the proof of the first part of the theorem is complete.

Suppose now that $\chi_{n}(A)=\sum_{\lambda \vdash n} m_{\lambda} \chi_{\lambda}$ and $m_{\lambda}=0$ whenever $|\lambda|-\lambda_{1} \geq q$. By [BR] the multiplicities $m_{\lambda}$ are polynomially bounded; hence

$$
c_{n}(A)=\sum_{\substack{\lambda \vdash n \\|\lambda|-\lambda_{1}<q}} m_{\lambda} d_{\lambda} \leq C n^{t} \sum_{\substack{\lambda \vdash n \\|\lambda|-\lambda_{1}<q}} d_{\lambda}
$$

and the proof now follows from the hook formula for the degrees $d_{\lambda}$.

The previous theorem says that $A$ has polynomial growth of the codimensions if and only if all the irreducible characters appearing with non-zero multiplicity in $\chi_{n}(A)$ have associated diagram with at most $q-1$ boxes below the first row where $J^{q}=0$.

\section{§3. Finite dimensional algebras with involution}

In this section we shall prove that if $A$ is a finite dimensional algebra with involution $*$, then in the decomposition $A=B+J$ we can choose $B$ to be invariant under *. Beside its own interest, this result will be used in the next section. Throughout we shall assume that $\operatorname{char} F \neq 2$. 
Theorem 4. Let $A$ be a finite dimensional algebra with involution $*$ over $F$ and $J$ its Jacobson radical. Then $J^{*}=J$ and there exists a maximal semisimple subalgebra $B$ such that $B=B^{*}$ and $A=B+J$.

Proof. It is obvious that $J^{*}=J$. Let $A=B+J$ with $B$ a semisimple subalgebra of $A$ and suppose first that $J^{2}=0$.

Since $B^{*}$ is also a maximal semisimple subalgebra of $A$, by the WedderburnMalcev theorem there exists $y \in J$ such that $B^{*}=(1-y) B(1+y)$. For $b \in B$ let $\bar{b} \in B$ be such that $b^{*}=(1-y) \bar{b}(1+y)$. Then $b=b^{* *}=\left(1+y^{*}\right) \bar{b}^{*}\left(1-y^{*}\right)=$ $\left(1+y^{*}\right)(1-y) \overline{\bar{b}}(1+y)\left(1-y^{*}\right)$, for a suitable $\overline{\bar{b}} \in B$. It follows that we can write $b=\overline{\bar{b}}+j$ for a suitable $j \in J$; hence $b-\overline{\bar{b}} \in B \cap J=0$ and $b=\overline{\bar{b}}$ follows. But then, from the above, $b=\left(1+y^{*}\right) \bar{b}^{*}\left(1-y^{*}\right)=\left(1+y^{*}\right)(1-y) b(1+y)\left(1-y^{*}\right)=$ $\left(1-y+y^{*}\right) b\left(1+y-y^{*}\right)$ since $J^{2}=0$. This says that $y-y^{*}$ commutes with $b$. Therefore by writing $y=\frac{y+y^{*}}{2}+\frac{y-y^{*}}{2}$, we get $b^{*}=(1-y) \bar{b}(1+y)=\left(1-\frac{y+y^{*}}{2}\right) \bar{b}\left(1+\frac{y+y^{*}}{2}\right)$.

We have proved that $B^{*}=(1-s) B(1+s)$ for a suitable symmetric element $s=s^{*} \in J$. At this stage it is easy to check that $B^{\prime}=\left(1-\frac{s}{2}\right) B\left(1+\frac{s}{2}\right)$ is the desired invariant subalgebra of $A$.

Suppose now that $J^{n}=0, J^{n-1} \neq 0, n>2$. Set $J^{n-1}=I$. Since $I^{*}=I, A / I$ has an induced involution; also $J(A / I)=J / I$ and $J(A / I)^{n-1}=0$. Therefore, by induction on $n, A / I=B / I+J / I$ for a suitable semisimple subalgebra $B / I=$ $(B / I)^{*}$. It follows that we can write $B=C+I$ where $C$ is a semisimple subalgebra of $B$ and, since $B^{*}=B$, by the first part we may assume that $C^{*}=C$. By counting dimensions we get that $C$ is a maximal semisimple subalgebra of $A$ and $A=C+J$ is the desired decomposition.

Recall that an algebra with involution $A$ is $*$-simple if $A$ has no proper *-invariant ideals (i.e., ideals $I$ such that $I^{*}=I$ ). It is well known and easy to prove that if $A$ is *-simple, then either $A$ is simple or $A \cong A_{1} \oplus A_{1}^{\text {op }}$ where $A_{1}$ is a simple homomorphic image of $A$ and $*$ on $A_{1} \oplus A_{1}^{\mathrm{op}}$ is the exchange involution $(a, b)^{*}=(b, a)$ (see [Ro. Proposition 2.13.24]).

Remark 1. If $B$ is a semisimple algebra with involution and $\operatorname{dim}_{F} B<\infty$, then $B=B_{1} \oplus \cdots \oplus B_{t}$ where $B_{1}, \ldots, B_{t}$ are $*$-simple algebras.

Proof. Let $B=C_{1} \oplus \cdots \oplus C_{m}$ be the decomposition of $B$ into simple components. Let $e_{1}, \ldots, e_{m}$ be the corresponding orthogonal central idempotents. Let $i \in\{1, \ldots, m\}$; if $e_{i}^{*}=e_{i}$, then $C_{i}=C_{i}^{*}=e_{i} B$ is $*$-simple. If $e_{i}^{*} \neq e_{i}$, then $e_{i}^{*} B$ is still a minimal ideal of $B$ which implies that $e_{i}^{*}=e_{j}$ for some $j \in\{1, \ldots, m\}$. Hence $C_{i} \oplus C_{j}$ is $*$-simple.

\section{§4. *-CODIMENSIONS WITH POLYNOMIAL GROWTH}

Throughout this section $F$ will be a field of characteristic zero, and $A$ an $F$ algebra with involution $*$. Let $A^{+}=\left\{a \in A \mid a=a^{*}\right\}$ and $A^{-}=\left\{a \in A \mid a=-a^{*}\right\}$ be the sets of symmetric and skew elements of $A$ respectively.

We consider $F\langle X, *\rangle=F\left\langle x_{1}, x_{1}^{*}, x_{2}, x_{2}^{*}, \ldots\right\rangle$ the free algebra with involution $*$ of countable rank. Recall that $f\left(x_{1}, x_{1}^{*}, \ldots, x_{n}, x_{n}^{*}\right) \in F\langle X, *\rangle$ is a $*$-polynomial identity for $A$ if $f\left(a_{1}, a_{1}^{*}, \ldots, a_{n}, a_{n}^{*}\right)=0$ for all $a_{1}, \ldots, a_{n} \in A$. The set $\operatorname{Id}(A, *)$ of all *-polynomial identities of $A$ is a T-ideal of $F\langle X, *\rangle$, i.e., an ideal invariant 
under all endomorphisms of $F\langle X, *\rangle$ commuting with the involution. Let

$$
V_{n}(*)=\operatorname{Span}_{F}\left\{x_{\sigma(1)}^{a_{1}} \cdots x_{\sigma(n)}^{a_{n}} \mid \sigma \in S_{n}, a_{i} \in\{1, *\}\right\}
$$

be the space of multilinear $*$-polynomials in $x_{1}, x_{1}^{*}, \ldots, x_{n}, x_{n}^{*}$.

If we set $s_{i}=x_{i}+x_{i}^{*}$ and $k_{i}=x_{i}-x_{i}^{*}, i=1,2, \ldots$, then, since $\operatorname{char} F \neq 2$, we can also write

$$
V_{n}(*)=\operatorname{Span}_{F}\left\{w_{\sigma(1)} \cdots w_{\sigma(n)} \mid \sigma \in S_{n}, w_{i}=s_{i} \text { or } w_{i}=k_{i}, i=1, \ldots, n\right\} .
$$

Let $H_{n}$ be the hyperoctahedral group. Recall that $H_{n}=\mathbb{Z}_{2} \sim S_{n}$ is the wreath product of $\mathbb{Z}_{2}=\{1, *\}$, the multiplicative group of order 2 , and $S_{n}$. We write the elements of $H_{n}$ as $\left(a_{1}, \ldots, a_{n} ; \sigma\right)$ where $a_{i} \in \mathbb{Z}_{2}, \sigma \in S_{n}$. The action of $H_{n}$ on $V_{n}(*)$ defined in GR] can be rewritten (see [DG]) as follows: for $h=\left(a_{1}, \ldots, a_{n} ; \sigma\right) \in H_{n}$ define $h s_{i}=s_{\sigma(i)}, h k_{i}=k_{\sigma(i)}^{a_{\sigma(i)}}= \pm k_{\sigma(i)}$ and then extend this action diagonally to $V_{n}(*)$. Hence $V_{n}(*)$ becomes a left $H_{n}$-module and, since $V_{n}(*) \cap I d(A, *)$ is a subspace invariant under this action, we can view $V_{n}(*) /\left(V_{n}(*) \cap I d(A, *)\right)$ as an $H_{n}$-module. Let $\chi_{n}(A, *)$ be its character.

The sequence $c_{n}(A, *)=\chi_{n}(A, *)(1)=\operatorname{dim}_{F} \frac{V_{n}(*)}{V_{n}(*) \cap I d(A, *)}, n=1,2, \ldots$, is called the sequence of $*$-codimensions of $A$.

Recall that there is a one-to-one correspondence between irreducible $H_{n}$-characters and pairs of partitions $(\lambda, \mu)$, where $\lambda \vdash r, \mu \vdash n-r$, for all $r=0,1, \ldots, n$. If $\chi_{\lambda, \mu}$ denotes the irreducible $H_{n}$-character corresponding to $(\lambda, \mu)$, then we can write

$$
\chi_{n}(A, *)=\sum_{r=0}^{n} \sum_{\substack{\lambda \vdash r \\ \mu \vdash n-r}} m_{\lambda, \mu} \chi_{\lambda, \mu}
$$

where $m_{\lambda, \mu} \geq 0$ are the corresponding multiplicities.

Now, for $r=0, \ldots, n$, we let

$$
\begin{aligned}
& V_{r, n-r}=\operatorname{Span}\left\{w_{\sigma(1)} \cdots w_{\sigma(n)} \mid \sigma \in S_{n}, w_{i}=s_{i} \text { for } i=1, \ldots, r\right. \text { and } \\
& \left.w_{i}=k_{i} \text { for } i=r+1, \ldots, n\right\} .
\end{aligned}
$$

Thus $V_{r, n-r}$ is the space of multilinear polynomials in $s_{1}, \ldots, s_{r}, k_{r+1}, \ldots, k_{n}$. It is clear that in order to study $V_{n}(*) \cap I d(A, *)$ it is enough to study $V_{r, n-r} \cap I d(A, *)$ for all $r$.

If we let $S_{r}$ act on the symmetric variables $s_{1}, \ldots, s_{r}$ and $S_{n-r}$ on the skew variables $k_{r+1}, \ldots, k_{n}$, we obtain an action of $S_{r} \times S_{n-r}$ on $V_{r, n-r}$ and

$$
V_{r, n-r}(A, *)=\frac{V_{r, n-r}}{V_{r, n-r} \cap \operatorname{Id}(A, *)}
$$

becomes a left $S_{r} \times S_{n-r}$-module. Let $\psi_{r, n-r}(A, *)$ be its character and

$$
c_{r, n-r}(A, *)=\psi_{r, n-r}(A, *)(1)=\operatorname{dim}_{F} V_{r, n-r}(A, *) .
$$

We write $\psi_{\lambda, \mu}$ for the irreducible $S_{r} \times S_{n-r}$-character associated to the pair $(\lambda, \mu)$ with $\lambda \vdash r, \mu \vdash n-r$. The following result holds.

Theorem 5 ([DG, Theorem 1.3]). Let $A$ be an algebra with involution; then, for all $n$,

$$
\chi_{n}(A, *)=\sum_{r=0}^{n} \sum_{\substack{\lambda \vdash r \\ \mu \vdash n-r}} m_{\lambda, \mu} \chi_{\lambda, \mu}, \quad \text { and } \quad \psi_{r, n-r}(A, *)=\sum_{\substack{\lambda \vdash r \\ \mu \vdash n-r}} m_{\lambda, \mu} \psi_{\lambda, \mu} .
$$


Moreover

$$
c_{n}(A, *)=\sum_{r=0}^{n}\left(\begin{array}{l}
n \\
r
\end{array}\right) c_{r, n-r}(A, *) .
$$

We next characterize finite dimensional algebras $A$ with polynomial growth of the $*$-codimensions.

Theorem 6. Let $A$ be a finite dimensional algebra with involution over an algebraically closed field $F$. Then the sequence of $*$-codimensions $\left\{c_{n}(A, *)\right\}_{n \geq 1}$ is polynomially bounded if and only if

1) the sequence of codimensions $\left\{c_{n}(A)\right\}_{n \geq 1}$ is polynomially bounded;

2) $A=B+J$, where $B$ is a maximal semisimple subalgebra of $A$ and $b=b^{*}$ for all $b \in B$.

Proof. By GR, Lemma 4.4], for all $n, c_{n}(A) \leq c_{n}(A, *) \leq \alpha n^{t}$ for some constants $\alpha, t$, and the sequence of codimensions is polynomially bounded. From [GZ it follows that $A=B+J$, where $B=B_{1} \oplus \cdots \oplus B_{m}$ and $B_{i} \cong F, B_{i} J B_{k}=0$ for all $i \neq k$. By Theorem 4 we may also assume that $B^{*}=B$.

Suppose by contradiction that $*$ is not the identity map on $B$; then, by Remark 1 , there exist $B_{i}, B_{k}$ such that $C=B_{i} \oplus B_{k} \cong F \oplus F$ is $*$-simple with involution $(a, b)^{*}=(b, a)$. Notice that, for all $\sigma \in S_{n}$ and $a_{1}, \ldots, a_{n} \in\{1, *\}$,

$$
x_{\sigma(1)}^{a_{\sigma(1)}} \cdots x_{\sigma(n)}^{a_{\sigma(n)}} \equiv x_{1}^{a_{1}} \cdots x_{n}^{a_{n}}(\bmod \operatorname{Id}(C, *)) .
$$

Moreover the set $\left\{x_{1}^{a_{1}} \cdots x_{n}^{a_{n}} \mid a_{i} \in\{1, *\}\right\}$ is linearly independent modulo $\operatorname{Id}(C, *)$. It follows that $c_{n}(C, *)=2^{n}$. Since $c_{n}(C, *) \leq c_{n}(A, *)$ we get a contradiction.

Suppose now that $A=B+J, c_{n}(A)$ is polynomially bounded and $*$ is the identity on $B$. In this case, if $a \in A$, write $a=b+j, b \in B, j \in J$. Then $a-a^{*}=j-j^{*} \in J$ and $A^{-} \subseteq J$.

Notice that if $f\left(x_{1}, \ldots, x_{n}\right) \in V_{n} \cap \operatorname{Id}(A)$, then, for every $r=0, \ldots, n$,

$$
f\left(s_{1}, \ldots, s_{r}, k_{r+1}, \ldots, k_{n}\right) \in V_{r, n-r} \cap \operatorname{Id}(A, *) .
$$

Hence $c_{r, n-r}(A, *) \leq c_{n}(A) \leq \alpha n^{t}$, for some $\alpha, t$, for all $r$. Let $J^{q}=0$. Since $A^{-} \subseteq J$, then, for all $r \leq n-q, V_{r, n-r} \cap I d(A, *)=V_{r, n-r}$ and $c_{r, n-r}(A, *)=0$ follows. By Theorem 5 for all $n$ we obtain

$$
\begin{gathered}
c_{n}(A, *)=\sum_{r=0}^{n}\left(\begin{array}{l}
n \\
r
\end{array}\right) c_{r, n-r}(A, *) \leq \alpha n^{t} \sum_{r=n-q+1}^{n}\left(\begin{array}{l}
n \\
r
\end{array}\right) \\
=\alpha n^{t} \sum_{r=0}^{q-1}\left(\begin{array}{l}
n \\
r
\end{array}\right) \leq \alpha n^{t+q}
\end{gathered}
$$

and $c_{n}(A, *)$ is polynomially bounded.

Next we want to get an analogue of Theorem 3 above by using the representation theory of the hyperoctahedral group $H_{n}$. We write $\chi_{\lambda, \mu}(1)=d_{\lambda, \mu}$ for the degree of the irreducible $H_{n}$-character $\chi_{\lambda, \mu}$. Recall that if $\lambda \vdash n, \mu \vdash n-r$, then $d_{\lambda, \mu}=$ $\left(\begin{array}{l}n \\ r\end{array}\right) d_{\lambda} d_{\mu}($ see $[\mathrm{DG}])$. 
Theorem 7. Let $A$ be a finite dimensional algebra with involution over a field $F$. Then the sequence of $*$-codimensions $\left\{c_{n}(A, *)\right\}_{n \geq 1}$ is polynomially bounded if and only if

$$
\chi_{n}(A, *)=\sum_{\substack{|\lambda|+|\mu|=n \\ n-\lambda_{1}<q}} m_{\lambda, \mu} \chi_{\lambda, \mu}
$$

where $J(A)^{q}=0$.

Proof. Since the decomposition of $V_{n}(*)$ into irreducible $H_{n}$-modules does not change by extending the scalars, as in the proof of Theorem 3 we may assume that $F$ is algebraically closed.

Suppose that the $*$-codimensions of $A$ are polynomially bounded and let $\lambda=$ $\left(\lambda_{1}, \ldots, \lambda_{t}\right) \vdash r, \mu \vdash n-r$ be such that $n-\lambda_{1} \geq q$. Suppose by contradiction that $m_{\lambda, \mu} \neq 0$; then there exist tableaux $T_{\lambda}, T_{\mu}$ such that $e_{T_{\lambda}} e_{T_{\mu}}$ has a non-trivial action on $V_{r, n-r}(A, *)$. This says that there exists a non trivial polynomial $f \in$ $e_{T_{\lambda}} e_{T_{\mu}} V_{r, n-r}$ such that $f=f\left(s_{1}, \ldots, s_{r}, k_{r+1}, \ldots, k_{n}\right)$ is not a $*$-identity of $A$.

We have that $f$ is a linear combination of polynomials each alternating on $t$ disjoint sets of $\lambda_{1}^{\prime}, \ldots, \lambda_{t}^{\prime}$ symmetric variables respectively. Let $g$ be one such polynomial; it is clear that, in order to finish the proof, it is enough to show that $g \equiv 0$ in $A$.

Since $B_{i} B_{k}=B_{i} J B_{k}=0$ for all $i \neq k$, we get $g \equiv 0$ on $A$ unless we substitute for the symmetric variables elements from one simple component, say $B_{i}$, and from $J$. Also, since $\operatorname{dim} B_{i}=1$, only one element of $B_{i}$ can be replaced for a variable in each alternating set. Thus, since $A^{-} \subseteq J$, in all we substitute at least $|\lambda|-\lambda_{1}+|\mu|=n-\lambda_{1} \geq q$ elements from $J$. Since $J^{q}=0$ we get that $g \equiv 0$ also in this case and the proof of the first part is complete.

Suppose now that $\chi_{n}(A, *)=\sum_{|\lambda|+|\mu|=n, n-\lambda_{1}<q} m_{\lambda, \mu} \chi_{\lambda, \mu}$. By a result of Berele ( $\mathrm{B}$, Theorem 15]) the multiplicities $m_{\lambda, \mu}$ are polynomially bounded. By recalling that if $|\lambda|-\lambda_{1}$ is bounded by a constant, then $d_{\lambda}$ is polynomially bounded, we get

$$
\begin{gathered}
c_{n}(A, *)=\sum_{\substack{|\lambda|+|\mu|=n \\
n-\lambda_{1}<q}} m_{\lambda, \mu} d_{\lambda, \mu} \leq \alpha n^{t} \sum_{\substack { r=n-q \\
\begin{subarray}{c}{\lambda \vdash r, \mu \vdash n-r \\
n-\lambda_{1}<q{ r = n - q \\
\begin{subarray} { c } { \lambda \vdash r , \mu \vdash n - r \\
n - \lambda _ { 1 } < q } }\end{subarray}}^{n}\left(\begin{array}{l}
n \\
r
\end{array}\right) d_{\lambda} d_{\mu} \\
\leq \alpha_{1} n^{t_{1}} \sum_{r=0}^{q}\left(\begin{array}{l}
n \\
r
\end{array}\right) \leq \alpha_{1} n^{t_{1}} n^{q+1} .
\end{gathered}
$$

The previous theorem says that a finite dimensional algebra with involution $A$ has polynomial growth of the $*$-codimensions if and only if all the irreducible $H_{n^{-}}$ characters $\chi_{\lambda, \mu}$ appearing with non-zero multiplicity in $\chi_{n}(A, *)$ are such that the diagram of $\lambda$, without the first row, and the diagram of $\mu$ contain in all at most $q$ boxes.

\section{REFERENCES}

[BR] A. Berele and A. Regev, Applications of hook diagrams to P.I. algebras, J. Algebra 82 (1983), 559-567. MR 84g:16012

[B] A. Berele, Cocharacter sequences for algebras with Hopf algebra actions, J. Algebra 185 (1996), 869-885. MR 97h:16032 
[CR] C. W. Curtis and I. Reiner, Representation Theory of Finite Groups and Associative Algebras, John Wiley and Sons, New York, 1962. MR 90g:16001

[DG] V. Drensky and A. Giambruno, Cocharacters, codimensions and Hilbert series of the polynomial identities for $2 \times 2$ matrices with involution, Canadian J. Math. 46 (1994), 718-733.

[GR] A. Giambruno and A. Regev, Wreath products and P.I. algebras, J. Pure Applied Algebra 35 (1985), 133-149. MR 86e:16027

[GZ] A. Giambruno and M. Zaicev, On codimension growth of finitely generated associative algebras, Adv. Math. 140 (1998), 145-155. CMP 99:05

[K1] A. Kemer, T-ideals with power growth of the codimensions are Specht, Sibirskii Matematicheskii Zhurnal 19 (1978), 37-48 (Russian), English transl Siberian Math. J.

[K2] A. Kemer, Ideals of identities of associative algebras, Transl. Math. Monogr., vol. 87, Amer. Math. Soc., Providence RI, 1988. MR 92f:16031

[KR] A. Krakowsky and A. Regev, The polynomial identities of the Grassmann algebra, Trans. AMS 181 (1973), 429-438. MR 48:4005

[R] A. Regev, Existence of identities in $A \otimes B$, Israel J. Math. 11 (1972), 131-152. MR 47:3442

[Ro] L. H. Rowen, Ring Theory, Academic Press, New York, 1988. MR 89h:16001

Dipartimento di Matematica e Applicazioni, Università di Palermo, Via Archirafi 34, 90123 PALERmo, ItAly

E-mail address: a.giambruno@unipa.it

Department of Algebra, Faculty of Mathematics and Mechanics, Moscow State UniVERsity, Moscow, 119899 Russia

E-mail address: zaicev@nw.math.msu.su 